\title{
Delayed Progression of Lung Metastases Following Delivery of a Prodrug-activating Enzyme
}

\author{
LOUIS DORE-SAVARD ${ }^{1}$, ZHIHANG CHEN ${ }^{1}$, PAUL T. WINNARD JR. ${ }^{1}$, BALAJI KRISHNAMACHARY ${ }^{1}$, \\ VENU RAMAN $^{1}$, MARGARET E. BLACK ${ }^{2}$ and ZAVER M. BHUJWALLA ${ }^{1}$ \\ ${ }^{1}$ Division of Cancer Imaging Research, The Russell H. Morgan Department of Radiology and Radiological Science, \\ The Johns Hopkins University School of Medicine, Baltimore, MD, U.S.A.; \\ ${ }^{2}$ School of Molecular Biosciences, Washington State University, Pullman, WA, U.S.A.
}

\begin{abstract}
Background: Chemotherapy is an effective option to treat recurrent or metastatic cancer but its debilitating sideeffects limit the dose and time of exposure. Prodrugs that can be activated locally by an activating enzyme can minimize collateral damage from chemotherapy. We previously demonstrated the efficacy of a poly-L-lysine-based theranostic nanoplex containing bacterial cytosine deaminase $(b C D)$ that locally converted 5-fluorocytosine (5-FC) to the chemotherapeutic agent 5-fluorouracil in MDA-MB-231 primary tumor xenografts. Materials and Methods: Here we used a more effective variant of $b C D$ to target metastatic red fluorescence protein expressing MDA-MB-435 cells in the lungs. We used an intravenous injection of tumor cells and monitored tumor growth in the lungs for 5 weeks by which time metastatic nodules were detected with optical imaging. The animals were then treated with the bCD-nanoplex and 5-FC. Results: We observed a significant decrease in metastatic burden with a single dose of the enzyme-nanoplex and two consecutive prodrug injections. Conclusion: These results are a first step towards the longitudinal evaluation of such a strategy with multiple doses. Additionally, the enzyme can be directly coupled to imaging reporters to time prodrug administration for the detection and treatment of aggressive metastatic cancer.
\end{abstract}

Despite significant improvements in the treatment of primary cancer, successful treatment of metastatic disease remains a challenge. Indeed, the 5-year survival rate for patients with

This article is freely accessible online.

Correspondence to: Zaver M. Bhujwalla, Johns Hopkins University School of Medicine, Traylor Building 208C, 720 Rutland Avenue, Baltimore, MD, 21205, U.S.A. Tel: +1 4109559698, Fax: +1 4106141948, e-mail: zaver@mri.jhu.edu

Key Words: Metastasis, prodrug enzyme therapy, imaging, cytosine deaminase, lung cancer. tumors distant from the primary site has not significantly improved in the past decade (1). The search for an alternative to systemic chemotherapy has thus been a priority, especially in the context of metastases.

Prodrug/enzyme treatments have been used to circumvent some side-effects of chemotherapy $(2,3)$. One of the enzymes that has met with some success is bacterial cytosine deaminase (bCD) from Escherichia coli. bCD converts the non-toxic prodrug 5-fluorocytosine (5-FC) to 5-fluorouracil (5-FU) (4). The latter potent chemotherapeutic agent has been used for decades in therapy of colorectal, head and neck, and breast cancer, among others. It is, however, associated with debilitating side-effects such as cardiotoxicity, diarrhea and myelosuppression (5) since high doses of systemic 5-FU are required to achieve significant tumor concentrations. To minimize sideeffects of 5-FU, targeted delivery of bCD to tumor cells coupled with systemic administration of 5-FC is also being considered. Monoclonal antibodies conjugated to the enzyme, as well as viral vectors and non-viral vectors, have been used to achieve this goal (6-13). Recent studies have demonstrated prostate cancer cell-specific nanoparticle delivery of bCD to tumor xenografts by specific targeting of prostate-specific membrane antigen that resulted in improved tumor control $(13,14)$ following localized conversion of 5-FC to 5-FU.

However, many types of cancer do not express cancer cell-specific surface receptors or antigens, making it important to determine the effect of 5-FU formed from bCD in the absence of targeted delivery. The therapeutic effect of $\mathrm{bCD} / 5-\mathrm{FC}$ in a metastatic disease setting remains sparsely evaluated (15-17). Our purpose in this study was to evaluate $\mathrm{bCD} / 5-\mathrm{FC}$ treatment in the metastatic setting.

Several mutants have been created for an enhanced conversion rate of 5-FC to 5-FU (18-20). A triple-mutant $\left(\mathrm{bCD}_{1525}\right)$ displaying high specificity for 5-FC (21) exhibited increased antitumor activity and also bystander effect, a phenomenon by which the cytotoxic metabolite is transferred to nearby cells via gap junction intercellular communications $(22,23)$. Here we used the $\mathrm{bCD}_{1525}$ triple-mutant enzyme to 
maximize the ability to reduce metastatic tumor burden. bCD was incorporated into a poly-L-lysine (PLL) nanoplex to achieve efficient delivery to the tumor tissue (24). We used aggressive metastatic MDA-MB-435 triple-negative breast cancer cells that expressed tandem dimer (td) Tomato red fluorescent protein to detect lung growth following intravenous injection of cancer cells.

\section{Materials and Methods}

Synthesis of the $b C D-P L L$ conjugate. Briefly, the synthesis of the bCD-PLL conjugate was performed by functionalizing PLL with $S$ acetylthioacetate and hydrazine to give multiple linker-labeled PLL. Branched polyethylenimine (PEI) was labeled with polyethyl glycol (PEG) $(2.0 \mathrm{kDa})$. The cografted PEI-PEG polymer was then labeled with benzaldehyde as a linker. Conjugation of equivalent functionalized PLL and PEI-PEG polymer resulted in the PLL-PEI copolymer. The $S$-acetylthioacetate in this copolymer was reduced to a sulfhydryl group for further reaction. This sulfhydryl group reacted with the $N$-[e-maleimidocaproyloxy]succinimide ester that was labeled to bCD to form the bCD-PLL-PEI nanocarrier.

Mouse model of lung metastases. All in vivo studies were carried out in compliance with institutional guidelines established by the Institutional Animal Care and Use Committee of Johns Hopkins University. MDA-MB-435 cells (American Type Culture Collection, Manassas, VA, USA) were inoculated at a density of $2 \times 10^{6}$ cells per $50 \mu 1$ Hank's balanced salt solution in the tail vein of female severe combined immunodeficient (SCID) mice (The Jackson Laboratory, Bar Harbor, ME, USA). The tumor cells expressed the fluorescent protein tdTomato as described previously (25). Tumors were detected in the lungs using in vivo imaging within 21 to 28 days, with large foci in both lungs observed at 28-35 days, at which time treatment was initiated (see below). Mice were anesthetized with a mixture of inhaled isoflurane (5\% for induction and $1.5-2 \%$ for maintenance) and oxygen during all injection and imaging procedures.

In vivo and ex vivo optical imaging studies. In vivo and ex vivo optical images were acquired with a Xenogen IVIS Spectrum scanner (Perkin-Elmer, Waltham, MA, USA), and images were processed by using Living Image 4.0 software (Caliper, Hopkinton, MA, USA). For in vivo optical imaging of the distribution of tdTomato from MDA-MB-435 cells, images were obtained at 3, 4 and 5 weeks after cancer cell injection and during week 5 prior to bCD administration. Mice were sacrificed at 3, 4, 5 and 7 weeks after cell injection for ex vivo imaging studies, and tumors and lymph nodes were excised to obtain the optical images. tdTomato fluorescence images were acquired using $\lambda_{\mathrm{ex}}=535-570$ and $\lambda_{\text {em }}=620-640 \mathrm{~nm}$ filter set, $1 \mathrm{~s}$ exposure time, and the fluorescence intensity expressed as photons $/ \mathrm{s} / \mathrm{cm}^{2} / \mathrm{sr}$.

Metastatic growth inhibition study. Five weeks after cancer cell inoculation and once tumor growth in the lungs was confirmed using optical imaging, mice were randomly divided in two groups (six mice per group) and treatments were applied as a) bCD nanoplex $(300 \mathrm{mg} / \mathrm{kg}$ i.v.), followed by $5-\mathrm{FC}(450 \mathrm{mg} / \mathrm{kg}$ i.p. and i.v.) 24 and $72 \mathrm{~h}$ later; or b) bCD nanoplex (300 mg/kg i.v.), followed by phosphate-bufferd saline (i.v. and i.p.) 24 and $72 \mathrm{~h}$ later. Tumor growth in the lungs was monitored for an additional 2 weeks prior to lung excision.

Histology. After mice were euthanized with a lethal i.v. dose of ketamine/acerpromazine, freshly inflated lungs were collected and examined under a Nikon TS100 microscope (Nikon Instruments, Melville, NY, USA) equipped with a digital camera microscope (magnification 10-40x). The tissue was then placed in a plastic cassette and fixed in $4 \%$ buffered formaldehyde solution for $72 \mathrm{~h}$. Fixed tissue was embedded in paraffin and blocks were cut in $3 \mu \mathrm{m}-$ thick sections. To observe cancer cell infiltration and tumor burden, non-adjacent sections were stained with Harris' hematoxylin and eosin, and images were captured by scanning the sections at high resolution on an Aperio ScanScope (Leica Biosystems Inc., Buffalo Grove, IL, USA). Metastatic burden was quantified by determining cancer cell-invaded portions of the lung using the ImageScope software version 12.1 (Leica Biosystems, Richmond, IL, USA) and normalizing the cancer cell-containing area to the total lung section area. Two non-consecutive sections per animal were analyzed and averaged for graphic representation.

Statistical analyses. Statistical analyses of tumor burden data were performed using a two-sided unpaired $t$-test using Graphpad Prism 5 (Graphpad, La Jolla, CA, USA). A p-value of 0.05 or less was considered significant.

\section{Results}

Enzyme/prodrug treatment reduces in vivo appearance of lung metastases. Fluorescent metastatic foci were detected between 3 to 4 weeks following cancer cell inoculation (Figure 1A). The number of foci and maximum fluorescence intensity increased over time and were widespread in both lungs by week 5 . A single dose of bCD-nanoplex followed by two injections of 5-FC delayed the progression of metastases at week 7 , as shown by decreased distribution of the signal in the treated group $(n=5)$ compared to the vehicletreated group $(n=6)$ (Figure 1B).

Cytosine deaminase nanoplex plus 5-FC reduces tumor burden in metastatic breast cancer. Qualitative ex vivo examination of lungs confirmed the in vivo observations (Figure 2A and B). We visually examined fluorescence from tdTomato-labelled MDA-MB-435 cancer cells and observed multiple nodules in lungs from both the bCD/5-FC-treated and control groups. However, larger metastatic tumors were detected in the vehicle-treated group.

Decreased metastatic tumor burden was confirmed by histology (Figure 2C and D). Cancer cells occupied a large fraction of the lungs in the control group. A representative section demonstrates large merged foci (Figure 2C) surrounded by several smaller cancer cell clusters. Extensive necrosis was also observed in the tumor foci of the control animals. Lungs from the treated group had smaller isolated clusters (Figure 2D), with a significant reduction of metastatic burden, as shown in Figure 3. 


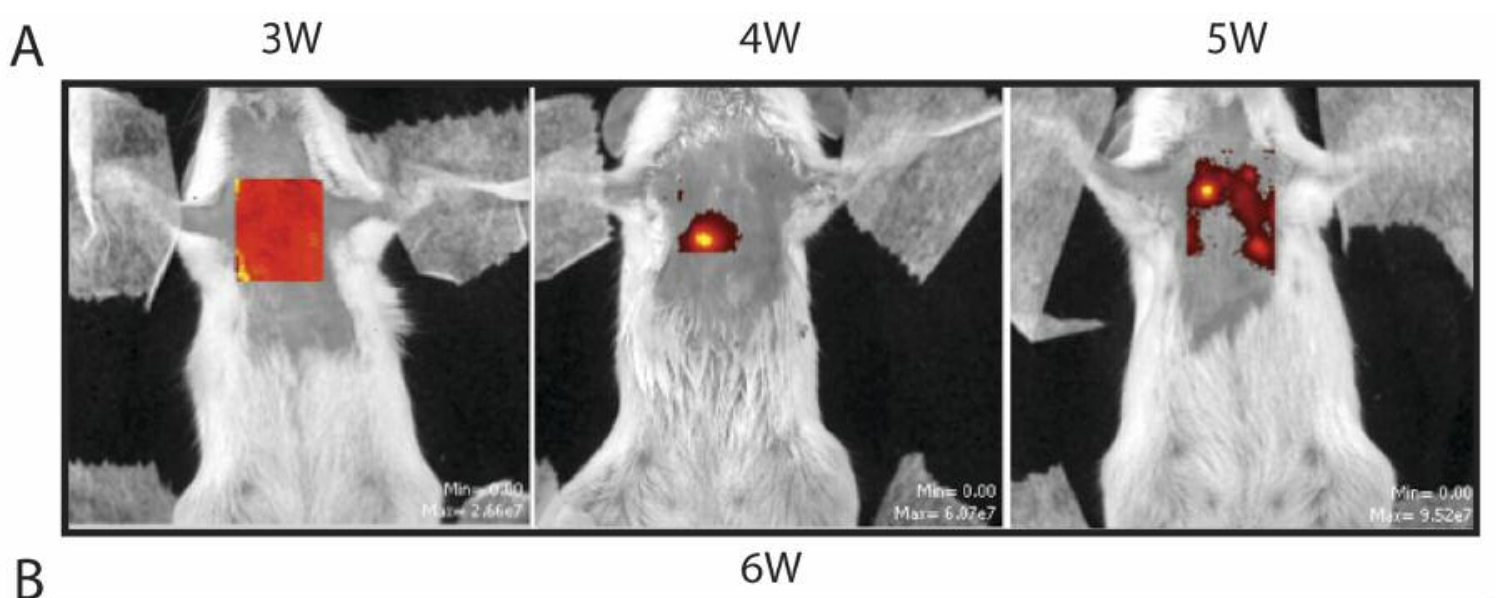

B

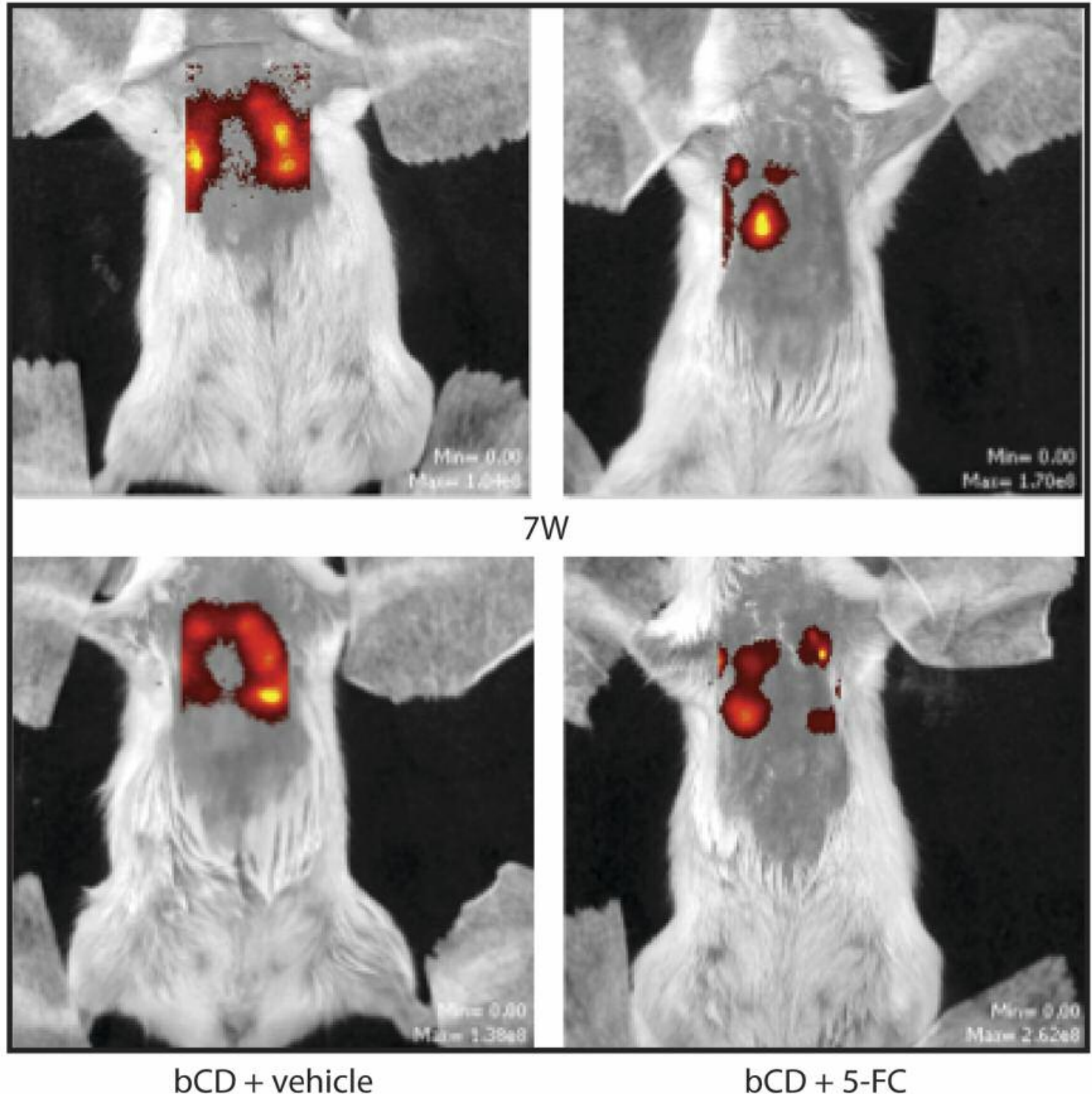

Figure 1. Lung metastases development in severe combined immunodeficient mice. Three weeks following the intravenous injection of MDA-MB435 cells, tumors remain undetectable. By weeks 4 and 5 , single or multiple nodules were detected and treatment was initiated (bacterial cytosine deaminase $(b C D)$ plus phosphate-buffered saline $(P B S)$ for the control group, $b C D$ plus 5-fluorocytosine for the treated group; $n=5-6)$. Treated animals still presented metastatic nodules but showed delayed progression of tumors at weeks 6 and 7. 

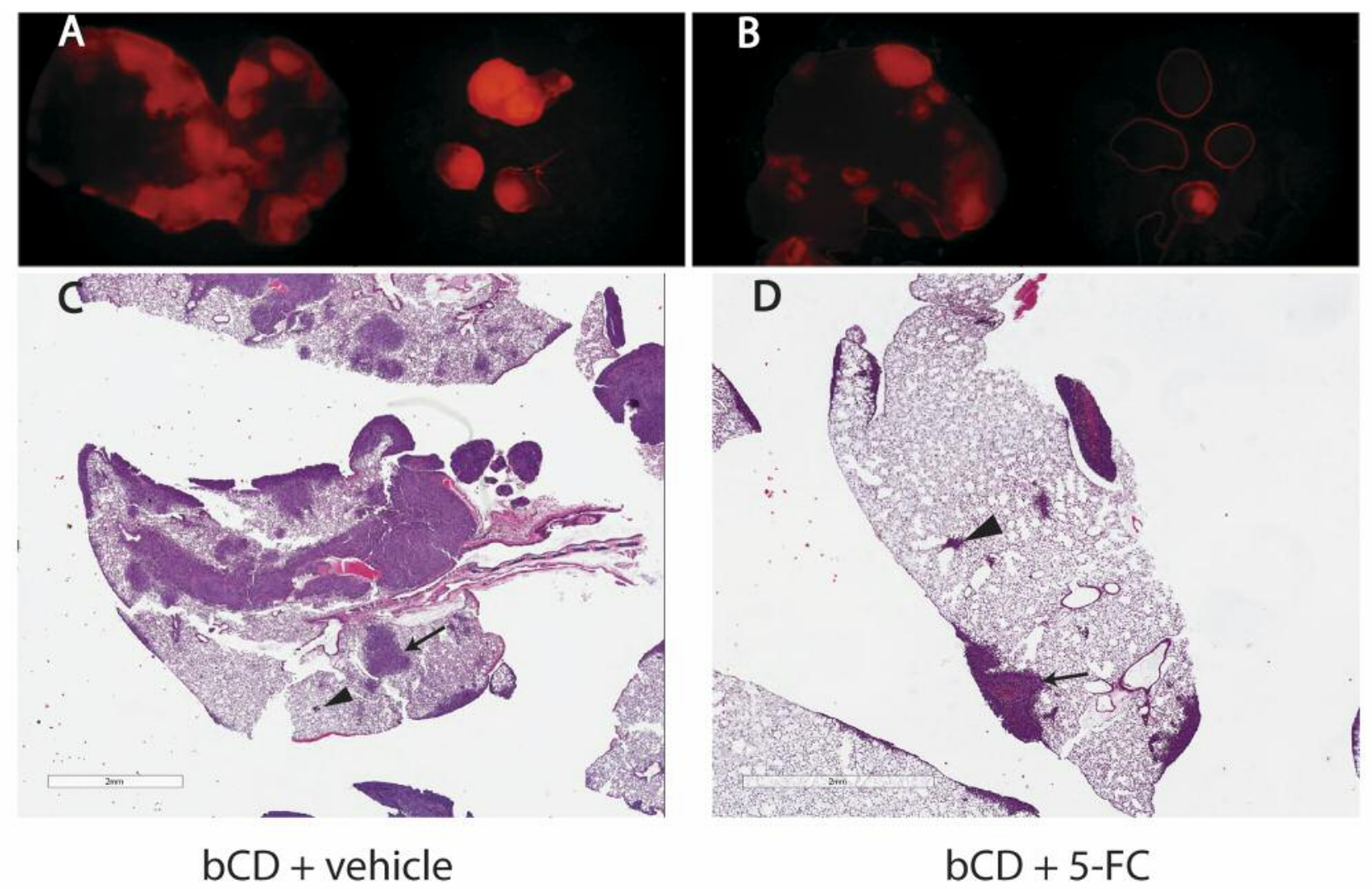

Figure 2. Ex vivo optical imaging $(A, B)$ and histological $(C, D)$ evaluation of metastatic tumor burden. After 10-14 days of bacterial cytosine deaminase $(b C D)$ plus 5-fluorocytosine injection, qualitative evaluation of the lungs and lymph nodes showed decreased tumor burden in treated animals ( $n=6)$ compared to controls $(n=5)$. Gross examination of the hematoxylin and eosin-stained sections also shows that lungs from both groups present macroscopic nodules (arrows) and micro-metastases (arrowheads). However, the treated group (D) shows reduced areas occupied by cancer cells.

\section{Discussion}

The reduction of the side-effects of systemic chemotherapy, and control of metastatic breast cancer can be achieved by local production of the chemotherapy agent within the metastatic lesion. Here we evaluated the effect of nontargeted delivery of bCD to metastatic lung tumors and observed a significant reduction of metastatic burden following a single administration of the enzyme followed by two sequential doses of 5-FC injected 24 and $72 \mathrm{~h}$ after bCD delivery. We previously showed that bCD is preferentially internalized by highly malignant cells, remains fully active after $24 \mathrm{~h}$ and that this delay represents the optimal time point to administer the prodrug (26). A single injection of the enzyme was sufficient to induce a significant decrease in growth despite the treatment being initiated at a relatively late stage of the disease, when multiple tumors were clearly detected in all animals. It would be interesting to determine if early administration of $\mathrm{bCD} / 5-\mathrm{FC}$ would be even more effective in reducing the metastatic burden. Here we tested
bCD/5-FC using MDA-MB-435 cells injected intravenously that primarily localize in the lungs. Our data support expanded evaluation of these studies with different metastasis models such as brain, bone, and other organs, as well as different cancer cell types.

Prodrug/enzyme systems have been investigated for nearly three decades (4). The expression of CD, in presence of 5FC, not only induced cell death and a bystander effect (27, 28), but also led to radiosensitization in various cancer models $(29,30)$. Breton and colleagues performed a study using a modified Ankara virus to express yeast $\mathrm{CD}$ in situ, reducing tumor growth in a glioblastoma mouse model (10). After introducing a viral vector for in vitro transformation of several cancer cell lines $(12,31)$ and evaluation in preclinical models (32), Tocagen is currently testing the Toca 511 virus for introducing CD into cancer cells in a phase II/III clinical trial in recurrent high-grade glioma (NCT02414165). The outcome of the clinical trial will provide data on effectiveness and potential side-effects, including the immunogenicity of such treatments. Previous studies have 


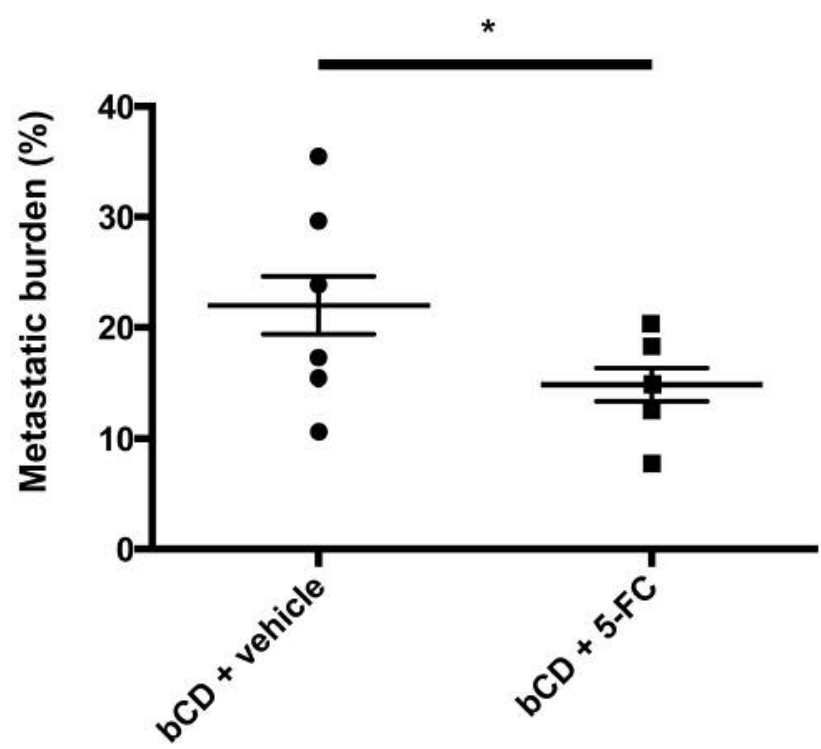

Figure 3. Quantitative evaluation of tumor burden in the lungs. Summary of metastatic burden derived from area occupied by cancer cells to the total lung area. Values represent the mean \pm SEM from six treated and five control mice. ${ }^{*} p<0.05$.

not detected significant immunogenicity of bCD in animals $(33,34)$. We do not anticipate that bCD $_{1525}$ would have immunogenic properties different from those of the regular protein, but this should be evaluated before translation to human use.

While designing cancer cell-specific targeting of the enzyme would be ideal and feasible for primary tumors where careful analysis of biopsy specimen is performed, this is not possible with metastatic disease. The nontargeted PLL conjugate we used in this study facilitates extravasation and has prolonged circulation time in vivo (26), allowing accumulation within tumors but not in normal tissue due to its size $(\mathrm{MW} \approx 350 \mathrm{kDa})$. Incorporation of a positron emission tomography/single photon emission computed tomography reporter within the nanoplex would allow for image-guided therapy of metastatic cancer as the prodrug can be administered once the presence of the enzyme at the lesion and its clearance from normal tissue is established. Here we have demonstrated that in the absence of cancer cell-specific targeting, it was possible to significantly reduce metastatic burden.

\section{Conclusion}

Patients affected by metastatic cancer rely mostly on aggressive systemic chemo- and radiotherapy for treatment. Here we demonstrated the potential use of systemic bCD/5-
FC for the treatment of lung metastases. A single treatment with the enzyme followed by two doses of the prodrug significantly delayed the growth of lung tumors in SCID mice. This proof-of-principle study supports the expanded evaluation of $\mathrm{bCD} / 5-\mathrm{FC}$ for control of metastatic disease.

\section{Acknowledgements}

LDS is a recipient of the Eileen-Iwanicki Fellowship for Breast Cancer Research, in collaboration with the Canadian Institutes of Health Research and the Breast Cancer Society of Canada. This work was supported by NIH R01CA138515 and R01CA82337. The Authors thank Ms Flonne Wildes for expert technical assistance.

\section{References}

1 Siegel RL, Miller KD and Jemal A: Cancer statistics, 2015. CA Cancer J Clin 65: 5-29, 2015.

2 Siegel R, Naishadham D and Jemal A: Cancer statistics, 2012. CA Cancer J Clin 62: 10-29, 2012.

3 Karjoo Z, Chen X and Hatefi A: Progress and problems with the use of suicide genes for targeted cancer therapy. Adv Drug Deliv Rev 99(Pt A): 113-128, 2016.

4 Nishiyama T, Kawamura Y, Kawamoto K, Matsumura H, Yamamoto N, Ito $\mathrm{T}$, Ohyama $\mathrm{A}$, Katsuragi $\mathrm{T}$ and Sakai $\mathrm{T}$ : Antineoplastic effects in rats of 5-fluorocytosine in combination with cytosine deaminase capsules. Cancer Res 45: 1753-1761, 1985.

5 Papanastasopoulos P and Stebbing J: Molecular basis of 5fluorouracil-related toxicity: lessons from clinical practice. Anticancer Res 34: 1531-1535, 2014.

6 Wallace PM, MacMaster JF, Smith VF, Kerr DE, Senter PD and Cosand WL: Intratumoral generation of 5-fluorouracil mediated by an antibody-cytosine deaminase conjugate in combination with 5-fluorocytosine. Cancer Res 54: 2719-2723, 1994.

7 Aboagye EO, Artemov D, Senter PD and Bhujwalla ZM: Intratumoral conversion of 5-fluorocytosine to 5-fluorouracil by monoclonal antibody-cytosine deaminase conjugates: noninvasive detection of prodrug activation by magnetic resonance spectroscopy and spectroscopic imaging. Cancer Res 58: 40754078, 1998.

8 Neves JV, Wilson JM and Rodrigues PNS: Transferrin and ferritin response to bacterial infection: the role of the liver and brain in fish. Dev Comp Immunol 33: 848-857, 2009.

9 Zhang G, Liu T, Chen Y-H, Chen Y, Xu M, Peng J, Yu S, Yuan $\mathrm{J}$ and Zhang $\mathrm{X}$ : Tissue specific cytotoxicity of colon cancer cells mediated by nanoparticle-delivered suicide gene in vitro and in vivo. Clin Cancer Res 15: 201-207, 2009.

10 Breton E, Goetz C, Kintz J, Accart N, Aubertin G, Grellier B, Erbs $\mathrm{P}$, Rooker, Constantinesco $\mathrm{A}$ and Choquet $\mathrm{P}$ : In vivo preclinical low-field MRI monitoring of tumor growth following a suicide-gene therapy in an orthotopic mice model of human glioblastoma. C. R. Biologies 333: 220-225, 2010.

11 Funaro MG, Nemani KV, Chen Z, Bhujwalla ZM, Griswold KE and Gimi B: Effect of alginate microencapsulation on the catalytic efficiency and in vitro enzyme-prodrug therapeutic efficacy of cytosine deaminase and of recombinant E. coli expressing cytosine deaminase. J Microencapsul 33(1): 64-70, 2016. 
12 Twitty CG, Diago OR, Hogan DJ, Burrascano C, Ibanez CE, Jolly DJ and Ostertag D: Retroviral replicating vectors deliver cytosine deaminase leading to targeted 5-fluorouracil-mediated cytotoxicity in multiple human cancer types. Hum Gene Ther Methods 27: 17-31, 2016.

13 Chen Z, Penet M-F, Krishnamachary B, Banerjee SR, Pomper MG and Bhujwalla ZM: PSMA-specific theranostic nanoplex for combination of TRAIL gene and 5-FC prodrug therapy of prostate cancer. Biomaterials 80: 57-67, 2016.

14 Chen Z, Penet M-F, Nimmagadda S, Li C, Banerjee SR, Winnard PT, Artemov D, Glunde K, Pomper MG and Bhujwalla ZM: PSMA-targeted theranostic nanoplex for prostate cancer therapy. ACS Nano 6: 7752-7762, 2012.

15 Nanni P, De Giovanni C, Nicoletti G, Landuzzi L, Rossi I, Frabetti F, Giovarelli M, Forni G, Cavallo F, Di Carlo E, Musiani P and Lollini PL: The immune response elicited by mammary adenocarcinoma cells transduced with interferongamma and cytosine deaminase genes cures lung metastases by parental cells. Hum Gene Ther 9: 217-224, 1998.

16 Gnant MF, Noll LA, Irvine KR, Puhlmann M, Terrill RE, Alexander HR and Bartlett DL: Tumor-specific gene delivery using recombinant vaccinia virus in a rabbit model of liver metastases. J Natl Cancer Inst 91: 1744-1750, 1999.

17 Zhang Z, Yin L, Zhang Y and Zhao F: In situ transduction of cytosine deaminase gene followed by systemic use of 5fluorocytosine inhibits tumor growth and metastasis in orthotopic prostate cancer mouse models. Chin Med J 115: 227231, 2002.

18 Mahan SD, Ireton GC, Stoddard BL and Black ME: Alaninescanning mutagenesis reveals a cytosine deaminase mutant with altered substrate preference. Biochemistry 43: 8957-8964, 2004.

19 Kaliberov SA, Market JM, Gillespie GY, Krendelchtchikova V, Manna Della D, Sellers JC, Kaliberova LN, Black ME and Buchsbaum DJ: Mutation of Escherichia coli cytosine deaminase significantly enhances molecular chemotherapy of human glioma. Gene Ther 14: 1111-1119, 2007.

20 Kaliberova LN, Manna Della DL, Krendelchtchikova V, Black ME, Buchsbaum DJ and Kaliberov SA: Molecular chemotherapy of pancreatic cancer using novel mutant bacterial cytosine deaminase gene. Mol Cancer Ther 7: 2845-2854, 2008.

21 Fuchita M, Ardiani A, Zhao L, Serve K, Stoddard BL and Black ME: Bacterial cytosine deaminase mutants created by molecular engineering show improved 5-fluorocytosine-mediated cell killing in vitro and in vivo. Cancer Res 69: 4791-4799, 2009.

22 Sato T, Neschadim A, Nakagawa R, Yanagisawa T and Medin JA: Evaluation of bystander cell killing effects in suicide gene therapy of cancer: engineered thymidylate kinase (TMPK)/AZT enzyme-prodrug axis. In: Methods in Molecular Biology. New York, NY, Springer New York, pp. 55-67, 2015.

23 Johnson AJ, Ardiani A, Sanchez-Bonilla M and Black ME: Comparative analysis of enzyme and pathway engineering strategies for 5-FC-mediated suicide gene therapy applications. Cancer Gene Ther 18: 533-542, 2011.

24 Li C, Winnard PT, Takagi T, Artemov D and Bhujwalla ZM: Multimodal image-guided enzyme/prodrug cancer therapy. J Am Chem Soc 128: 15072-15073, 2006.
25 Winnard PT, Kluth JB and Raman V: Noninvasive optical tracking of red fluorescent protein-expressing cancer cells in a model of metastatic breast cancer. Neoplasia 8: 796-806, 2006.

26 Li C, Wildes F, Winnard P, Artemov D, Penet M-F and Bhujwalla ZM: Conjugation of poly-L-lysine to bacterial cytosine deaminase improves the efficacy of enzyme/prodrug cancer therapy. J Med Chem 51: 3572-3582, 2008.

27 Li Z, Shanmugam N, Katayose D, Huber B, Srivastava S, Cowan K and Seth P: Enzyme/prodrug gene therapy approach for breast cancer using a recombinant adenovirus expressing Escherichia coli cytosine deaminase. Cancer Gene Ther 4: 113117, 1997.

28 Pierrefite-Carle V, Baque P, Gavelli A, Benchimol D, Bourgeon A, Milano G, Saint-Paul MC and Rossi B: Regression of experimental liver tumor after distant intra-hepatic injection of cytosine deaminase-expressing tumor cells and 5-fluorocytosine treatment. Int J Mol Med 5: 275-278, 2000.

29 Hanna NN, Mauceri HJ, Wayne JD, Hallahan DE, Kufe DW and Weichselbaum RR: Virally directed cytosine deaminase/5fluorocytosine gene therapy enhances radiation response in human cancer xenografts. Cancer Res 57: 4205-4209, 1997.

30 Hamstra DA, Rice DJ, Pu A, Oyedijo D, Ross BD and Rehemtulla A: Combined radiation and enzyme/prodrug treatment for head and neck cancer in an orthotopic animal model. Radiat Res 152: 499-507, 1999.

31 Ostertag D, Amundson KK, Lopez Espinoza F, Martin B, Buckley T, Galvão da Silva AP, Lin AH, Valenta DT, Perez OD, Ibanez CE, Chen C-I, Pettersson PL, Burnett R, Daublebsky V, Hlavaty J, Gunzburg W, Kasahara N, Gruber HE, Jolly DJ and Robbins JM: Brain tumor eradication and prolonged survival from intratumoral conversion of 5-fluorocytosine to 5fluorouracil using a nonlytic retroviral replicating vector. NeuroOncology 14: 145-159, 2012.

32 Huang TT, Parab S, Burnett R, Diago O, Ostertag D, Hofman FM, Espinoza FL, Martin B, Ibanez CE, Kasahara N, Gruber HE, Pertschuk D, Jolly DJ and Robbins JM: Intravenous administration of retroviral replicating vector, Toca 511, demonstrates therapeutic efficacy in orthotopic immunecompetent mouse glioma model. Hum Gene Ther 26: 82-93, 2015.

33 Stella V, Borchardt R, Hageman M, Oliyai R, Maag H, Tilley J (Eds.) : Prodrugs: Challenges and Rewards. Springer, 2007.

34 Li C, Penet M-F, Wildes F, Takagi T, Chen Z, Winnard PT, Artemov D and Bhujwalla ZM: Nanoplex delivery of siRNA and prodrug enzyme for multimodality image-guided molecular pathway targeted cancer therapy. ACS Nano 4: 6707-6716, 2010.

Received March 8, 2017

Revised March 27, 2017

Accepted March 31, 2017 